\title{
ERROR ESTIMATES OF A DIFFERENCE APPROXIMATION METHOD FOR A BACKWARD HEAT CONDUCTION PROBLEM
}

XIANG-TUAN XIONG, CHU-LI FU, ZHI QIAN, AND XIANG GAO

Received 22 March 2005; Revised 11 December 2005; Accepted 12 February 2006

We introduce a central difference method for a backward heat conduction problem (BHCP). Error estimates for this method are provided together with a selection rule for the regularization parameter (the space step length). A numerical experiment is presented in order to illustrate the role of the regularization parameter.

Copyright (c) 2006 Hindawi Publishing Corporation. All rights reserved.

\section{Introduction}

The backward heat conduction problem (BHCP) is also referred to as the final boundary value problem. In general no solution which satisfies the heat conduction equation with final data and the boundary conditions exists. Even if a solution exists, it will not be continuously dependent on the final data. The BHCP is a typical example of an illposed problem which is unstable in numerical simulations and requires special regularization methods. In the context of approximation method for this problem, many approaches have been investigated. Such authors as Lattès and Lions [6], Showalter [10], Ames [1], Miller [9] have approximated the BHCP by quasi-reversibility methods. In [11], Schröter and Tautenhahn established an optimal error estimate for a special BHCP. Mera and Jourhmane used many numerical methods with regularization techniques to approximate the problem in $[8,5]$, and so forth. A mollification method has been studied by Hào in [4]. Recently, Liu [7] used a group preserving scheme to solve the backward heat equation numerically. A difference approximation method for solving sideways heat equation was provided by Eldén in [2], where he used a central difference to replace the time derivative in heat equation. In this paper, we consider a special BHCP [4]:

$$
\begin{gathered}
u_{t}(x, t)=u_{x x}(x, t), \quad x \in \mathbb{R}, 0<t<1, \\
u(x, 1)=\varphi(x), \quad x \in \mathbb{R} .
\end{gathered}
$$

We want to obtain the temperature distribution $u(x, t)$ for $0<t<1$. Since the data $\varphi(\cdot)$ are based on (physical) observations and are not known with complete accuracy, we assume 
2 Error estimates for a backward heat conduction problem

that $\varphi(\cdot)$ and $\varphi^{\delta}(\cdot)$ satisfy

$$
\left\|\varphi(\cdot)-\varphi^{\delta}(\cdot)\right\| \leq \delta
$$

where $\varphi(\cdot)$ and $\varphi^{\delta}(\cdot)$ belong to $L^{2}(\mathbb{R}), \varphi^{\delta}(\cdot)$ denotes the measured data and $\delta$ denotes the noise level. As usual, assume that there exists an a priori condition for our problem (1.1):

$$
\|u(\cdot, 0)\| \leq E,
$$

where $E$ is a positive bound. Problem (1.1) has a unique solution according to [3]. In order to use the Fourier transform technique, we define the Fourier transform of function $f(x)(x \in \mathbb{R})$ as the following:

$$
\hat{f}(\xi):=\frac{1}{\sqrt{2 \pi}} \int_{-\infty}^{\infty} e^{-i x \xi} f(x) d x, \quad \xi \in \mathbb{R} .
$$

We consider the problem (1.1) in $L^{2}$-space with respect to the variable $x$. Then taking Fourier transform with respect to $x$, the problem (1.1) can be reformulated in frequency space as follows:

$$
\widehat{u}_{t}(\xi, t)=(i \xi)^{2} \widehat{u}(\xi, t), \quad \widehat{u}(\xi, 1)=\hat{\varphi}(\xi), \quad \xi \in \mathbb{R} .
$$

The solution to (1.5) is given by

$$
\widehat{u}(\xi, t)=e^{\xi^{2}(1-t)} \widehat{\varphi}(\xi) .
$$

Then by inverse Fourier transform, the unique solution of (1.1) can be expressed:

$$
u(x, t)=\frac{1}{\sqrt{2 \pi}} \int_{-\infty}^{\infty} e^{i x \xi} e^{\xi^{2}(1-t)} \hat{\varphi}(\xi) d \xi
$$

From (1.6), we can easily see that

$$
\widehat{u}(\xi, 0)=e^{\xi^{2}} \hat{\varphi}(\xi)
$$

Since in our problem $\hat{u}(\xi, t)$ is assumed to be in $L^{2}(\mathbb{R})$, we see that the exact data function $\hat{\varphi}(\xi)$ must decay rapidly as $\xi \rightarrow \infty$. In the same time, it is easy to see that a small perturbation in the data $\hat{\varphi}(\xi)$ may cause dramatically large error in the solution $\hat{u}(\xi, t)$. In addition, the magnifying factor is $e^{\xi^{2}}$; hence, it is a severely ill-posed problem. By using central difference with step length $h$ to approximate the second derivative $u_{x x}$, we can get a regularized problem (with noisy data):

$$
\begin{gathered}
v_{t}(x, t)=\frac{v(x+h, t)-2 v(x, t)+v(x-h, t)}{h^{2}}, \quad x \in \mathbb{R}, 0<t<1, \\
v(x, 1)=\varphi^{\delta}(x), \quad x \in \mathbb{R} .
\end{gathered}
$$

Similarly we can easily get a solution in the frequency space for problem (1.9):

$$
\widehat{v}(\xi, t)=e^{4 \sin ^{2}(h \xi / 2)(1-t) / h^{2}} \hat{\varphi}^{\delta}(\xi) .
$$


Then by inverse Fourier transform,

$$
v(x, t)=\frac{1}{\sqrt{2 \pi}} \int_{-\infty}^{\infty} e^{i x \xi} e^{4 \sin ^{2}(h \xi / 2)(1-t) / h^{2}} \hat{\varphi}^{\delta}(\xi) d \xi .
$$

In the forthcoming section, we will see that (1.11) is a stable approximate solution for the problem (1.1).

\section{Error estimates}

In this section, we will prove error estimates between the exact solution (1.7) and the approximate solution (1.11). The following conclusion is the main result of this paper.

Theorem 2.1. Supposed that $u(x, t)$ is given by (1.7) with exact data $\varphi$ and that $v(x, t)$ is given by (1.11) with noisy data $\varphi^{\delta}$. If there exists a bound $\|u(\cdot, 0)\| \leq E$ and the data functions satisfy $\left\|\varphi-\varphi^{\delta}\right\| \leq \delta$, and if $h=2(\ln (E / \delta))^{-1 / 2}$ is chosen, then the following error estimates can be obtained.

(I) If $E / \delta<e^{3 / 2}$, then for $0<t<1$,

$$
\|u(\cdot, t)-v(\cdot, t)\| \leq 2 E^{1-t} \delta^{t}
$$

(II) If $E / \delta \geq e^{3 / 2}$, then

(II-a) for a fixed $t$ satisfying $1 /(3 / 2) \ln (E / \delta)<t<1$,

$$
\|u(\cdot, t)-v(\cdot, t)\| \leq \max \left\{(1-t)\left(\ln \frac{E}{\delta}\right)^{-1 / 2}\left(\frac{3}{2 \mathrm{et}}\right)^{3 / 2}, E^{1-t} \delta^{t}\right\}+E^{1-t} \delta^{t},
$$

(II-b) for a fixed t satisfying $0<t \leq(1) /((3 / 2) \ln (E / \delta))$,

$$
\|u(\cdot, t)-v(\cdot, t)\| \leq \max \left\{\frac{1-t}{3}\left(\ln \frac{E}{\delta}\right) E^{1-t} \delta^{t}, E^{1-t} \delta^{t}\right\}+E^{1-t} \delta^{t} .
$$

Proof. By Parseval relation and (1.8), (1.3), and (1.2), we can get

$$
\begin{aligned}
\| u(\cdot, t) & -v(\cdot, t) \| \\
= & \|\widehat{u}(\cdot, t)-\hat{v}(\cdot, t)\|=\left\|e^{(1-t) \xi^{2}} \hat{\varphi}(\xi)-e^{(1-t)\left(4 \sin ^{2}(\xi h / 2) / h^{2}\right)} \hat{\varphi}^{\delta}(\xi)\right\| \\
\leq & \left\|e^{(1-t) \xi^{2}} \hat{\varphi}(\xi)-e^{(1-t)\left(4 \sin ^{2}(\xi h / 2) / h^{2}\right)} \hat{\varphi}(\xi)\right\| \\
& +\left\|e^{(1-t)\left(4 \sin ^{2}(\xi h / 2) / h^{2}\right)} \hat{\varphi}(\xi)-e^{(1-t)\left(4 \sin ^{2}(\xi h / 2) / h^{2}\right)} \hat{\varphi}^{\delta}(\xi)\right\| \\
= & \left\|e^{-t \xi^{2}} e^{\xi^{2}} \hat{\varphi}(\xi)-e^{(1-t)\left(4 \sin ^{2}(\xi h / 2) / h^{2}\right)} e^{-\xi^{2}} e^{\xi^{2}} \hat{\varphi}(\xi)\right\| \\
& +\left\|e^{(1-t)\left(4 \sin ^{2}(\xi h / 2) / h^{2}\right)} \hat{\varphi}(\xi)-e^{(1-t)\left(4 \sin ^{2}(\xi h / 2) / h^{2}\right)} \hat{\varphi}^{\delta}(\xi)\right\| \\
\leq & \sup _{\xi \in \mathbb{R}}\left|e^{-t \xi^{2}}-e^{(1-t)\left(4 \sin ^{2}(\xi h / 2) / h^{2}\right)-\xi^{2}}\right|\|\hat{u}(\xi, 0)\| \\
& +\sup _{\xi \in \mathbb{R}}\left|e^{(1-t)\left(4 \sin ^{2}(\xi h / 2) / h^{2}\right)}\right| \delta \leq \sup _{\xi \in \mathbb{R}} A(\xi) E+\sup _{\xi \in \mathbb{R}} B(\xi) \delta,
\end{aligned}
$$


4 Error estimates for a backward heat conduction problem

where

$$
\begin{gathered}
A(\xi):=\left|e^{-t \xi^{2}}-e^{(1-t)\left(4 \sin ^{2}(\xi h / 2) / h^{2}\right)-\xi^{2}}\right|, \\
B(\xi):=\left|e^{(1-t)\left(4 \sin ^{2}(\xi h / 2) / h^{2}\right)}\right| .
\end{gathered}
$$

Firstly we can estimate $B(\xi) \delta$ as

$$
B(\xi) \delta \leq e^{(1-t)\left(4 / h^{2}\right)} \delta
$$

According to the selection of $h$ in Theorem 2.1, we have

$$
B(\xi) \delta \leq e^{(1-t) \ln (E / \delta)} \delta=E^{1-t} \delta^{t} .
$$

Now we will devote to estimating $A(\xi) E$,

$$
\begin{aligned}
A(\xi) E & =\left|e^{-t \xi^{2}}-e^{(1-t)\left(4 \sin ^{2}(\xi h / 2) / h^{2}\right)-\xi^{2}}\right| E \\
& =e^{-t \xi^{2}}\left|1-e^{(1-t)\left(4 \sin ^{2}(\xi h / 2) / h^{2}-\xi^{2}\right)}\right| E .
\end{aligned}
$$

Obviously,

$$
\frac{4 \sin ^{2}(\xi h / 2)}{h^{2}} \leq \xi^{2} .
$$

Case 1. If $|\xi| \geq 2 / h$, hence

$$
e^{(1-t)\left(4 \sin ^{2}(\xi h / 2) / h^{2}-\xi^{2}\right)} \leq 1
$$

furthermore for the selection of $h$, we have

$$
A(\xi) E \leq e^{-t \xi^{2}} E \leq e^{-t\left(4 / h^{2}\right)} E=E^{1-t} \delta^{t} .
$$

Case 2. If $|\xi| \leq 2 / h$, from (2.8) and by the inequality $1-e^{-y} \leq y(y \geq 0)$, we have

$$
A(\xi) \leq e^{-t \xi^{2}}\left(\xi^{2}-\frac{4 \sin ^{2}(\xi h / 2)}{h^{2}}\right)(1-t) .
$$

Let $r:=h|\xi| / 2$, and note that $0 \leq r \leq 1, \xi^{2}=4 r^{2} / h^{2}$, we have

$$
\begin{aligned}
A(\xi) & \leq e^{-t\left(4 r^{2} / h^{2}\right)} \frac{1}{h^{2}}\left(4 r^{2}-4 \sin ^{2} r\right)(1-t) \\
& =e^{-t\left(4 r^{2} / h^{2}\right)} \frac{4}{h^{2}}(r+\sin r)(r-\sin r)(1-t) \\
& \leq 2 e^{-\operatorname{tr}^{2}\left(4 / h^{2}\right)} \frac{4}{h^{2}}(r-\sin r)(1-t) .
\end{aligned}
$$


By using the inequality $\sin r \geq r-\left(r^{3} / 6\right)(r \geq 0)$, we get

$$
A(\xi) \leq 2 e^{-\operatorname{tr}^{2}\left(4 / h^{2}\right)} \frac{4}{h^{2}} \frac{r^{3}}{6}(1-t):=Q(r)
$$

We can easily find that the uniqueness maximum of $Q(r)$ attains at the point $r_{0}=$ $\sqrt{(3 / 2 t)\left(h^{2} / 4\right)}=\sqrt{(3 / 2 t)(\ln E / \delta)^{-1}}$, then

$$
A(\xi) \leq Q_{\max }=Q\left(r_{0}\right)=(1-t)\left(\ln \frac{E}{\delta}\right)^{-1 / 2}\left(\frac{3}{2 t e}\right)^{3 / 2}, \quad \text { for } r_{0}<1
$$

If $r_{0}>1$, then maximum of $Q(r)$ is $Q(1)$, then

$$
A(\xi) \leq Q_{\max }=Q(1)=\frac{1-t}{3}\left(\ln \frac{E}{\delta}\right) E^{-t} \delta^{t} .
$$

From $r_{0}=\sqrt{(3 / 2)(1 / \ln (E / \delta) / t)}$, note that $0<t<1$, hence if $(3 / 2)(1 / \ln (E / \delta))>1$, that is, $\ln E / \delta<3 / 2$, then $r_{0}>1$. We can conclude that the following hold.

(I) If $(E / \delta)<e^{3 / 2}$, then $r_{0}>1$ is always satisfied. Hence for all $\xi \in \mathbb{R}$, combining (2.11) and (2.16) we have

$$
\begin{aligned}
\|\hat{u}(\cdot, t)-\hat{v}(\cdot, t)\| & \leq \sup _{\xi \in \mathbb{R}} A(\xi) E+\sup _{\xi \in \mathbb{R}} B(\xi) \delta \\
& \leq \max \left\{Q(1) E, E^{1-t} \delta^{t}\right\}+E^{1-t} \delta^{t} \\
& =\max \left\{\frac{(1-t)}{3}\left(\ln \frac{E}{\delta}\right) E^{1-t} \delta^{t}, E^{1-t} \delta^{t}\right\}+E^{1-t} \delta^{t} \\
& \leq \max \left\{\frac{(1-t)}{3} \frac{3}{2} E^{1-t} \delta^{t}, E^{1-t} \delta^{t}\right\}+E^{1-t} \delta^{t} \\
& \leq\left(\max \left\{\frac{(1-t)}{(2,1)}\right\}+1\right) E^{1-t} \delta^{t} \\
& \leq 2 E^{1-t} \delta^{t} \longrightarrow 0, \text { for } \delta \longrightarrow 0 .
\end{aligned}
$$

(II) If $E / \delta \geq e^{3 / 2}$, then

(II-a) if $1 /(3 / 2) \ln (E / \delta)<t<1$, then $r_{0} \leq 1$ holds, combining (2.11) and (2.15), we have

$$
\begin{aligned}
& \|\hat{u}(\cdot, t)-\widehat{v}(\cdot, t)\| \\
& \leq \sup _{\xi \in \mathbb{R}} A(\xi) E+\sup _{\xi \in \mathbb{R}} B(\xi) \delta \leq \max \left\{Q\left(r_{0}\right) E, E^{1-t} \delta^{t}\right\}+E^{1-t} \delta^{t} \\
& =\max \left\{(1-t)\left(\frac{3}{2 e t}\right)^{3 / 2}\left(\ln \frac{E}{\delta}\right)^{-1 / 2}, E^{1-t} \delta^{t}\right\}+E^{1-t} \delta^{t} \longrightarrow 0, \quad \text { for fixed } t, \delta \longrightarrow 0 ;
\end{aligned}
$$


6 Error estimates for a backward heat conduction problem

(II-b) if $0<t \leq 1 /(3 / 2) \ln (E / \delta)$, then $r_{0}>1$ holds, combining (2.11) and (2.16), we have

$$
\begin{aligned}
& \| \hat{u}(\cdot, t)-\hat{v}(\cdot, t) \| \\
& \leq \sup _{\xi \in \mathbb{R}} A(\xi) E+\sup _{\xi \in \mathbb{R}} B(\xi) \delta \leq \max \left\{Q(1) E, E^{1-t} \delta^{t}\right\}+E^{1-t} \delta^{t} \\
&=\max \left\{\frac{(1-t)}{3}\left(\ln \frac{E}{\delta}\right) E^{1-t} \delta^{t}, E^{1-t} \delta^{t}\right\}+E^{1-t} \delta^{t} \longrightarrow 0, \quad \text { for fixed } t \delta \rightarrow 0 .
\end{aligned}
$$

Thus, the proof of the theorem is completed.

It is easy to see that the space step length $h$ is the regularization parameter of this problem. In the conclusion, we give a rule for choosing the regularization parameter, which is very important for the study of ill-posed problems.

\section{A numerical example}

In this section, by a numerical experiment, we will study how the regularization parameter $h$ influences the approximation.

It is easy to verify that the function

$$
u(x, t)=\frac{1}{\sqrt{1+4 t}} e^{-x^{2} /(1+4 t)}
$$

is the unique solution of the initial problem

$$
\begin{aligned}
& u_{t}=u_{x x}, \quad x \in \mathbb{R}, t>0, \\
& u(x, 0)=e^{-x^{2}}, \quad x \in \mathbb{R} .
\end{aligned}
$$

Hence, $u(x, t)$ given by (3.1) is also the solution of the following backward heat equation for $0 \leq t<1$ :

$$
\begin{aligned}
& u_{t}=u_{x x}, \quad x \in \mathbb{R}, 0 \leq t<1, \\
& u(x, 1)=\frac{1}{\sqrt{5}} e^{-x^{2} / 5}, \quad x \in \mathbb{R} .
\end{aligned}
$$

Let $s=1-t, w(x, s)=u(x, t)$, we have

$$
\begin{aligned}
w_{s} & =-w_{x x}, \quad x \in \mathbb{R}, 0<s<1, \\
w(x, s=0) & =\frac{1}{\sqrt{5}} e^{-x^{2} / 5}, \quad x \in \mathbb{R} .
\end{aligned}
$$

If $w_{x x}$ at $x_{i}$ is replaced by a second-order central difference, then (3.4) becomes

$$
w_{s}\left(x_{i}, s\right)=-\frac{1}{h^{2}}\left[w\left(x_{i}+h, s\right)-2 w\left(x_{i}, s\right)+w\left(x_{i}-h, s\right)\right] .
$$


First let us list some notations: $x_{i}=i h$, for $i=-n, \ldots, 0, \ldots, n . w_{i}=w_{i}(s)=w(i h, s)$. Then (3.6) with the initial condition (3.5) can be discretized as

$$
\begin{aligned}
& \left(\begin{array}{c}
w_{-n} \\
\vdots \\
w_{0} \\
\vdots \\
w_{n}
\end{array}\right)_{s}=\left(\begin{array}{cccc}
\frac{2}{h^{2}} & -\frac{1}{h^{2}} & & 0 \\
-\frac{1}{h^{2}} & \frac{2}{h^{2}} & -\frac{1}{h^{2}} & \\
& \ddots & \ddots & \ddots \\
0 & & -\frac{1}{h^{2}} & \frac{2}{h^{2}}
\end{array}\right)_{(2 n+1) \times(2 n+1)}\left(\begin{array}{c}
w_{-n} \\
\vdots \\
w_{0} \\
\vdots \\
w_{n}
\end{array}\right), \\
& \left(\begin{array}{c}
w_{-n}(0) \\
\vdots \\
w_{0}(0) \\
\vdots \\
w_{n}(0)
\end{array}\right)=\left(\begin{array}{c}
\frac{1}{\sqrt{5}} e^{-x_{-n}^{2} / 5} \\
\vdots \\
\frac{1}{\sqrt{5}} e^{-x_{0}^{2} / 5} \\
\vdots \\
\frac{1}{\sqrt{5}} e^{-x_{n}^{2} / 5}
\end{array}\right) .
\end{aligned}
$$

This ordinary differential equation system can easily be solved. In our numerical implementation we use a Runge-Kutta method. A standard ODE solver ode45 in Matlab is such a method.

Now we focus on the numerical experiment. The main aim is to investigate the role of regularization parameter $h$. We do the numerical experiment in the interval $x \in[-20,20]$ and $t \in[0,1]$. This is reasonable in that the initial data at the points $x=-20,20$ in (3.5) can be considered to be 0 in the computation by noting that the final value $u(x, 1) \rightarrow 0$ in (3.3) when $x \rightarrow \pm \infty$. To test the accuracy of an approximate solution, we compute the root mean square error (RMSE) by

$$
E(u)=\sqrt{\frac{1}{2 n+1} \sum_{i=-n}^{n}\left(v_{i}-u_{i}\right)^{2}}
$$

at total $2 n+1$ test points at the $x$-axis, where $v_{i}$ and $u_{i}$ are, respectively, the approximate and exact temperature at a test point. Obviously, (3.9) is to approximate $L^{2}$ norm error. For convenience, in our numerical experiment the noisy data is generated by

$$
w_{i}^{\delta}(0)=w_{i}(0)+\delta, \quad i=-n, \ldots, n,
$$

where $w_{i}(0)$ are the exact data given in (3.8). Thus, Since (3.9), it follows $E(w(0))=\delta$.

From Figure 3.1, one can see that for fixed $\delta=0.001$, the $L^{2}$-error attain a minimum at the "optimal" $h=2(\ln (E / \delta))^{-1 / 2} \approx 0.76$ (here we take $\|u(x, 0)\|_{L^{2}(\mathbb{R})}=E \approx 1.1$ ).

From Figure 3.2, we can see that for a fixed $h$, the $L^{2}$-error increase as $t$ approaches 0 . 
8 Error estimates for a backward heat conduction problem

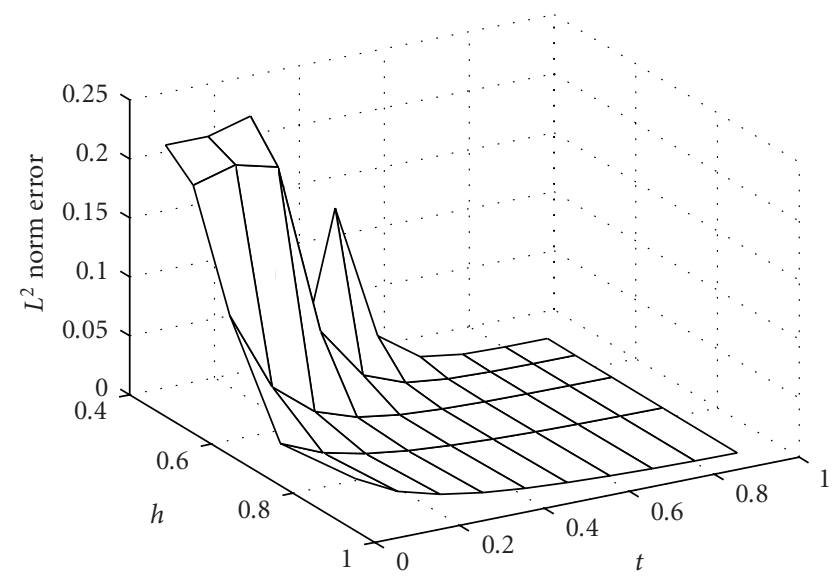

Figure 3.1. $L^{2}$-error for various $t$ and $h$ with fixed $\delta=0.001$ (“optimal” $h=10 / 13 \doteq 0.77$ ).

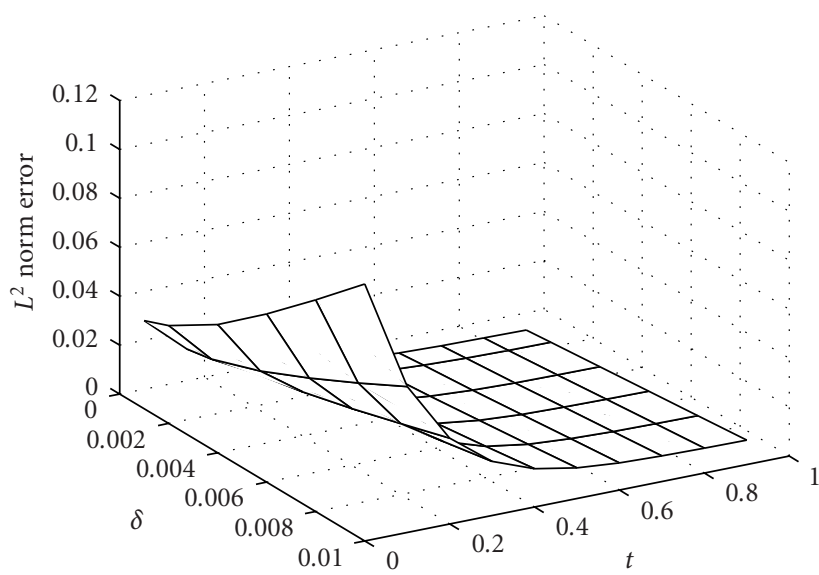

Figure 3.2. $L^{2}$-error for various $t$ and $\delta$ (for fixed $h=10 / 13$ ).

\section{Acknowledgments}

This work is supported by the National Natural Science Foundation of China (no. 10271050), the Natural Science Foundation of Gansu Province of China (no. 3ZS051A25-015), and the Fundamental Research Fund for Physics and Mathematics of Lanzhou University (no. Lzu05-05). The authors would like to thank the referees for their valuable comments and suggestions.

\section{References}

[1] K. A. Ames, G. W. Clark, J. F. Epperson, and S. F. Oppenheimer, A comparison of regularizations for an ill-posed problem, Mathematics of Computation 67 (1998), no. 224, 1451-1471. 
[2] L. Eldén, Numerical solution of the sideways heat equation by difference approximation in time, Inverse Problems 11 (1995), no. 4, 913-923.

[3] L. C. Evans, Partial Differential Equations, Graduate Studies in Mathematics, vol. 19, American Mathematical Society, Rhode Island, 1998.

[4] D. N. Hào, A mollification method for ill-posed problems, Numerische Mathematik 68 (1994), no. 4, 469-506.

[5] M. Jourhmane and N. S. Mera, An iterative algorithm for the backward heat conduction problem based on variable relaxtion factors, Inverse Problems in Engineering 10 (2002), no. 4, 293-308.

[6] R. Lattès and J.-L. Lions, Méthode de quasi-réversibilité et applications, Travaux et Recherches Mathématiques, no. 15, Dunod, Paris, 1967, English translation R. Bellman, Elsevier, New York, 1969.

[7] C. S. Liu, Group preserving scheme for backward heat conduction problems, International Journal of Heat and Mass Transfer 47 (2004), no. 12-13, 2567-2576.

[8] N. S. Mera, L. Elliott, L. D. B. Ingham, and D. Lesnic, An iterative boundary element method for solving the one dimensional backward heat conduction problem, International Journal of Heat and Mass Transfer 44 (2001), no. 10, 1973-1981.

[9] K. Miller, Stabilized quasi-reversibility and other nearly-best-possible methods for non-well-posed problems, Symposium on Non-Well-Posed Problems and Logarithmic Convexity (Heriot-Watt University, Edinburgh, 1972), Lecture Notes in Mathematics, vol. 316, Springer, Berlin, 1973, pp. 161-176.

[10] R. E. Showalter, The final value problem for evolution equations, Journal of Mathematical Analysis and Applications 47 (1974), 563-572.

[11] U. Tautenhahn and T. Schröter, On optimal regularization methods for the backward heat equation, Zeitschrift für Analysis und ihre Anwendungen 15 (1996), no. 2, 475-493.

Xiang-Tuan Xiong: Department of Mathematics, Lanzhou University,

Lanzhou 730000, China

E-mail address: xiongxt04@st.lzu.edu.cn

Chu-Li Fu: Department of Mathematics, Lanzhou University, Lanzhou 730000, China

E-mail address: fuchuli@lzu.edu.cn

Zhi Qian: Department of Mathematics, Lanzhou University, Lanzhou 730000, China

E-mail address: qianzh03@st.lzu.edu.cn

Xiang Gao: Department of Mathematics, Lanzhou University, Lanzhou 730000, China

E-mail address: gaoxiang03@st.lzu.edu.cn 


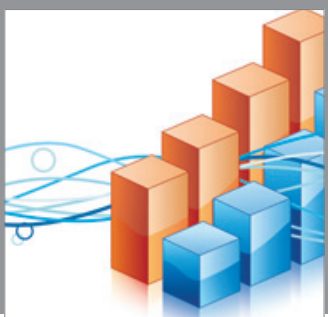

Advances in

Operations Research

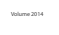

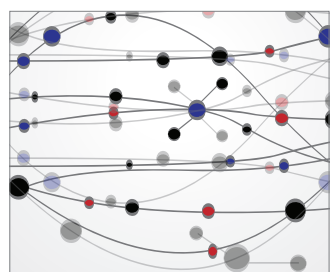

\section{The Scientific} World Journal
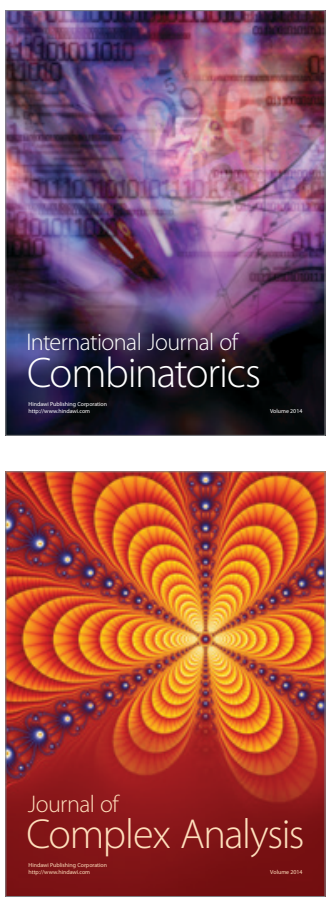

International Journal of

Mathematics and

Mathematical

Sciences
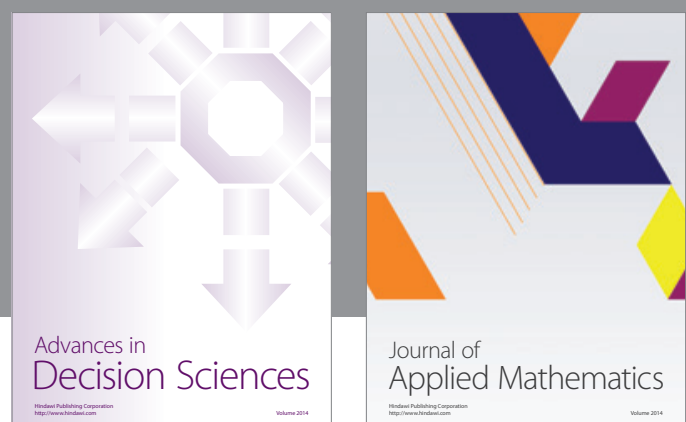

Journal of

Applied Mathematics
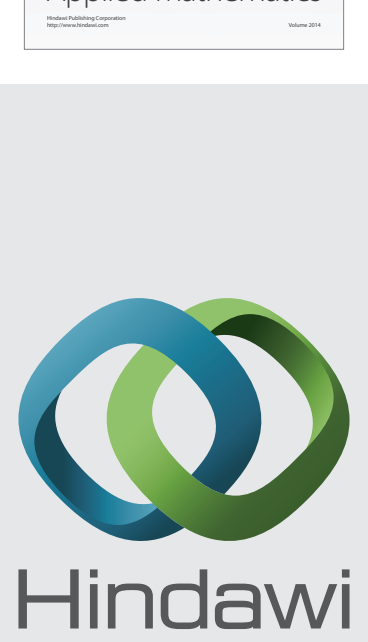

Submit your manuscripts at http://www.hindawi.com
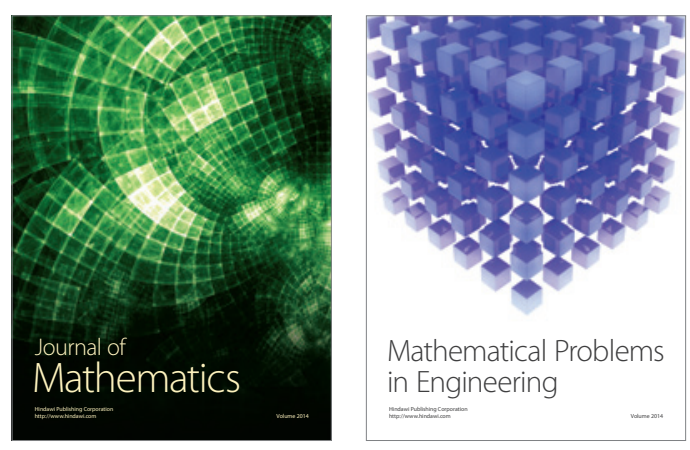

Mathematical Problems in Engineering
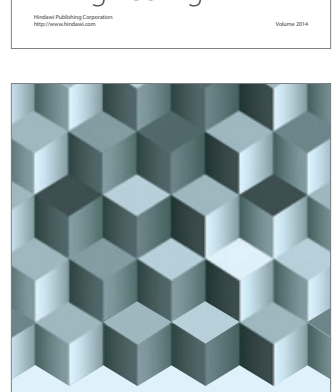

Journal of

Function Spaces
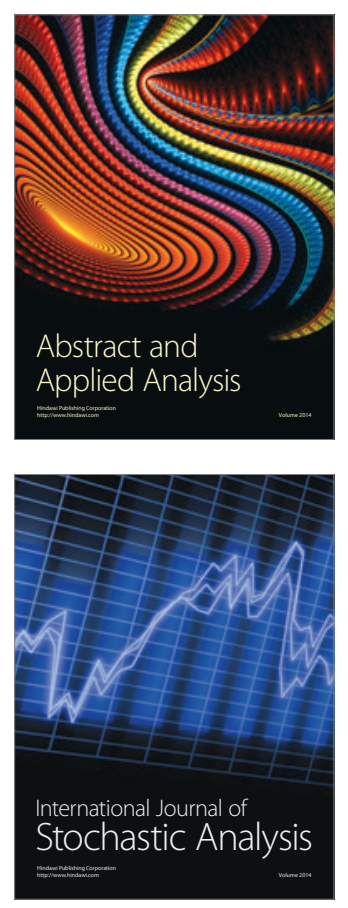

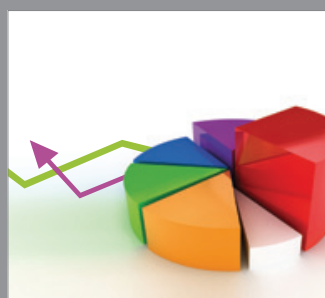

ournal of

Probability and Statistics

Promensencen
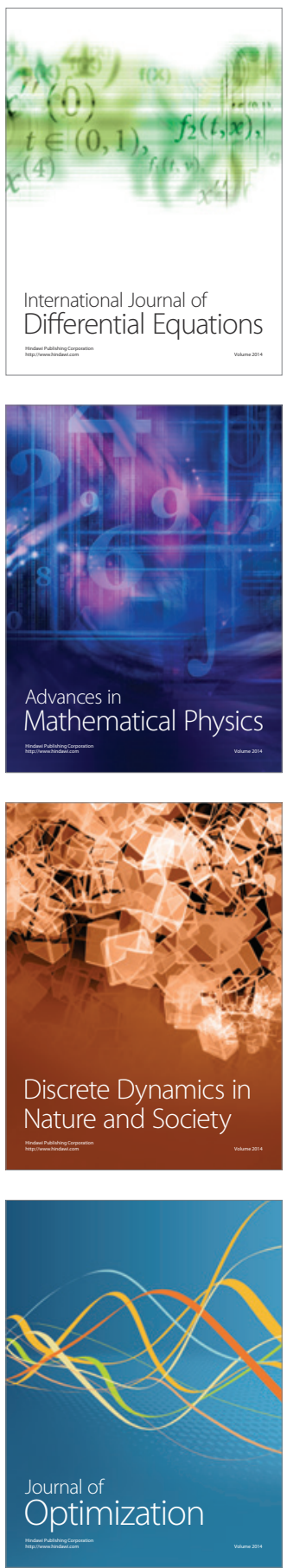